\title{
Analysis of Spatial Harmonics in a Polyphase Electrostatic Induction Micromotor
}

\author{
F. J. Santana-Martín, J. M. Monzón-Verona, S. García-Alonso, J. A. Montiel-Nelson
}

\begin{abstract}
This paper analyzes the influence on the force density of the spatial harmonics of the excitation voltage signal on each electrode of an electrostatic induction micromotor. We have used the lumped parametric equivalent circuit [7] and demonstrated that the spatial harmonics are attenuated from stator to rotor.
\end{abstract}

Index Terms-Electrostatic induction micromotor, spatial harmonics, parametric equivalent circuit.

\section{NOMENCLATURE}

\begin{tabular}{lll}
\hline Symbol & Name & Unit \\
\hline$a$ & Height of dielectric $a$, air & $(\mathrm{m})$ \\
$b$ & Height of dielectric $b$, rotor & $(\mathrm{m})$ \\
$C_{1}$ & Capacitance 1 & $(\mathrm{F} / \mathrm{m})$ \\
$C_{2}$ & Capacitance 2 & $(\mathrm{F} / \mathrm{m})$ \\
$G^{\prime}$ & Conductance 2 & $(\mathrm{S} / \mathrm{m})$ \\
$G_{r}^{\prime}$ & Variable Conductance & $(\mathrm{S} / \mathrm{m})$ \\
$k$ & Number of waves per metre & - \\
$j$ & Imaginary unity & - \\
$S$ & Slip & - \\
$T_{t}$ & Force density tangential component & $\left(\mathrm{N} / \mathrm{m}^{2}\right)$ \\
$v$ & Linear speed of mobile part & $(\mathrm{m} / \mathrm{s})$ \\
$V$ & Interelectrodic voltage & $(\mathrm{V})$ \\
$V_{0}$ & Supply voltage & $(\mathrm{V})$ \\
$V_{l}$ & Voltage in node 1 & $(\mathrm{V})$ \\
$V_{2}$ & Voltage in node 2 & $(\mathrm{V})$ \\
$\varepsilon_{a}$ & Permittivity of the dielectric $a$ & $(\mathrm{~F} / \mathrm{m})$ \\
$\varepsilon_{b}$ & Permittivity of the dielectric $b$ & $(\mathrm{~F} / \mathrm{m})$ \\
$\varepsilon_{e f f}$ & Effective permittivity & $(\mathrm{F} / \mathrm{m})$ \\
$\omega$ & Angular frequency of the signal & $(\mathrm{Hz})$ \\
$\sigma_{a}$ & Conductivity of the dielectric $a$ & $(\mathrm{~S} / \mathrm{m})$ \\
$\sigma_{b}$ & Conductivity of the dielectric $b$ & $(\mathrm{~S} / \mathrm{m})$ \\
$\sigma_{e f f}$ & Effective Conductivity & $(\mathrm{S} / \mathrm{m})$ \\
$\sigma_{S}$ & Surface Conductivity & $(\mathrm{S})$ \\
$\Phi^{b}$ & Voltage at the interface & $(\mathrm{V})$ \\
$\Psi$ & Objective function & - \\
\hline & &
\end{tabular}

\section{INTRODUCTION}

$\mathrm{E}$ lectrical micromachines, such as micromotors and microgenerators, have been fabricated thanks to the advance on material processing in the microscale and to the use of well-known techniques utilized in the microelectronic world [1]. These micromachines, called power MEMS, include devices such as gas microturbines, micropumps, microcompressors, among others. In this paper we study the distribution of the voltage spatial harmonics in an electrostatic induction micromotor, and its influence on the force density of the mobile part of the micromotor.

To perform the analysis we use the physical model of the micromotor. This model has been obtained from Maxwell's

F. J. Santana-Martín (e-mail: fsantana@die.ulpgc.es) and J. M Monzón-Verona (e-mail: jmonzon@die.ulpgc.es) are with the Institute for Applied Microelectronics and the Department of Electrical Engineering, University of Las Palmas de Gran Canaria, 35017-Las Palmas de Gran Canaria, Spain.

S. García-Alonso (e-mail: sgarcia@diea.ulpgc.es) and J. A. MontielNelson (e-mail: montiel@iuma.ulpgc.es) are with the Institute for Applied Microelectronics and the Department of Electronic Engineering and Automatic, University of Las Palmas de Gran Canaria, 35017-Las Palmas de Gran Canaria, Spain. equations [2]-[3], taking into account the boundary conditions of the micromotor. The boundary conditions of the stator of the micromachine, consist in a set of voltages applied to six electrodes — six conduction plates - with a time phase difference [1] and [4]. In this way, a traveling voltage wave that induces a charge on the mobile part is obtained. Mobile part and stator remain electrostatically coupled. The traveling voltage wave makes possible the movement of the mobile part, which causes the output mechanical power [5] and [6].

Published works dealing with induction micromotors obtain the torque from a pure sinusoidal voltage traveling wave associated to the power supply [7].

For the purpose of this work, we take into account all the harmonics of the travelling wave, and study their influence on the force density function. In addition, we use a simple equivalent circuit that helps to obtain the main magnitudes of interest in a micromotor, such as power consumption, force density, among others.

Fig. 1 illustrates the boundary conditions applied to the six conductive plates, from 1 to 6 . The maximum applied voltage is $200(\mathrm{~V})$.

To obtain the force densities for the different harmonics, from Maxwell's equations - Gauss theorem, continuity equation and constitutive equations - we obtained the electric field equation of the electrostatic induction micromotor [7]:

$$
\nabla \sigma \nabla \Phi+\frac{\partial}{\partial t} \nabla \varepsilon \nabla \Phi=0
$$

Equation (1) is a differential-algebraic system of equations (DAE) that is represented as:

$$
M \frac{\partial \Phi}{\partial t}+N \Phi=f(t)
$$

where $M$ and $N$ are matrix of coefficients, $\Phi$ is the potential at the interface, and $f(t)$ is the excitation potential signal on each electrode when Dirichlet conditions are used to solve the FEM equation system. This time-dependent equation has to be discretizated in both space and time domain. The space domain is discretized with triangular elements using Gmsh software [8]. The discretization in time domain has been realized applying the $\theta$-method. Then, (3) takes the following expression:

$$
M \frac{\Phi_{n+1}-\Phi_{n}}{\Delta t}+N\left(\theta \Phi_{n+1}+(1-\theta) \Phi_{n}\right)=\theta b_{n-1}+(1-\theta) b_{n}
$$

where index $n$ and $n+1$ refer to $\Phi$ quantities at time $t$ and $t^{t} \Delta t$, respectively. Depending on the values for parameter $\theta$, 
we obtain a particular equation that is solved by a classical method. For example, if $\theta=1$ we use implicit Euler method, when $\theta=0$ explicit Euler method and $\theta=0.5$ is CrankNicholson [9]. In this paper we apply $\theta=1$.

For the entire domain, at $t=0$, the initial conditions for the electric potential is $V=0$. The total time for the transient analysis is 14 cycles of the applied sinusoidal potential with a maximum value of $200(\mathrm{~V})$. The time step used in $\theta$ method is equal to $\mathrm{T} / 40(\mathrm{~s})$, where $\mathrm{T}$ is the signal period (see Table I). The potential at the interface has been calculated with the Finite Element Method (FEM), using the GetDP [10] software solution. At $t=0$ the initial condition for the electric potential is $\Phi=0$, in the entire domain.

TABLE I

PHYSICAL AND GEOMETRICAL PARAMETERS OF THE MiCROMACHINE

\begin{tabular}{|c|c|r|c|}
\hline Symbol & Name & \multicolumn{1}{|c|}{ Value } & Unit \\
\hline$L$ & Length of the structure & $44 \cdot 10^{-6}$ & $(\mathrm{~m})$ \\
\hline$h m$ & Height of the metallic plates & $0.01 \cdot 10^{-6}$ & $(\mathrm{~m})$ \\
\hline$a$ & Height of dielectric 2 & $3 \cdot 10^{-6}$ & $(\mathrm{~m})$ \\
\hline$b$ & Height of dielectric 1 & $10 \cdot 10^{-6}$ & $(\mathrm{~m})$ \\
\hline$k$ & Number of waves per metre & $2 \pi / \mathrm{L}$ & $\left(\mathrm{m}^{-1}\right)$ \\
\hline$v$ & Linear speed of mobile part & 0 & $(\mathrm{~m} / \mathrm{s})$ \\
\hline$f$ & Temporal frequency of excitation & $2.6 \cdot 10^{6}$ & $(\mathrm{~Hz})$ \\
\hline$V_{0}$ & Maximum value of excitation & 200 & $(\mathrm{~V})$ \\
\hline
\end{tabular}

The paper is organized as follows. Section III introduces the lumped parametric equivalent circuit model which is used to obtain the force density in the interface of the micromotor. Section IV explains in detail the spatial distribution of harmonics for the potential and the force density. Finally, conclusions and future works are presented in section $\mathrm{V}$.

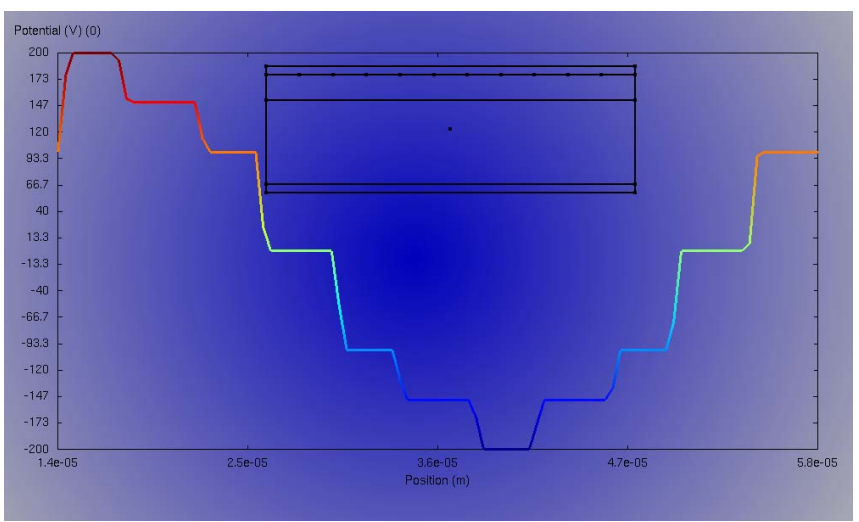

Fig. 1. Boundary conditions. Potential at the micromotor electrodes.

\section{LumPED PARAMETRIC EQUiVALENT CIRCUIT MODEL}

The quality of the circuit model [7] is based on how its magnitudes of interest match the main phenomena under study in the micromotor (force density, potential at the interface, etc.). The proposed lumped parametric model defines a micromotor in terms of circuit linear passive elements $\left(C_{1}, C_{2}, G_{2}^{\prime}\right.$, etc.) - electric field Equation (1) of the micromotor does not contain non-linear elements for a constant temperature - and a network or topological structure. Each element has its own constitutive equations that are expressed in a mathematical form. The network or topological structure defines the system configuration. The composition of these mathematical relations for each element gives us a system of algebraic equations.

Fig. 2(a) represents the micromotor physical model. Fig. 2(b) shows the proposed lumped parametric equivalent circuit model. As is shown in Fig. 2(a) and (b), the equivalence between the physical model and the proposed lumped parametric equivalent circuit model is straightforward.

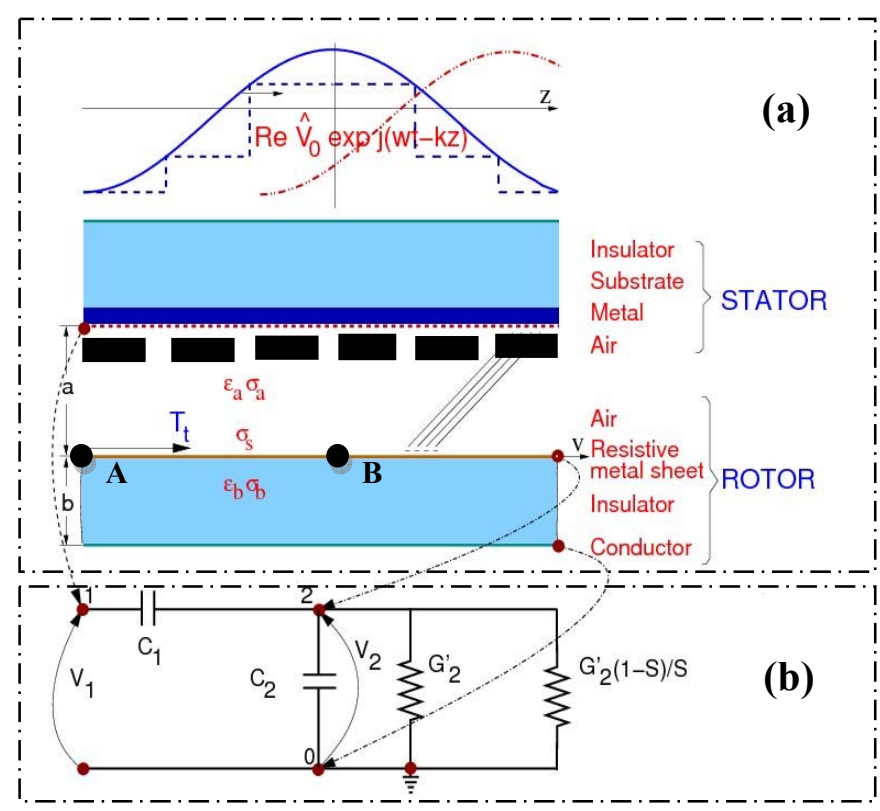

Fig. 2. a) Physical model and b) lumped parametric equivalent models for the electrostatic induction micromotor per face.

The proposed model explains and predicts the behavior of the electrostatic induction micromotor, without expensive temporal simulations, saving computational resources. To the best of our knowledge, no lumped parametric equivalent circuit model per phase has been published in the literature for the electrostatic induction micromotor.

In this parametric model for an electrostatic induction micromotor, there are not inductive impedances because they are neglected for second order effects. Instead, we introduce capacitive impedances that are characteristic of the physical nature of the micromotor. The correspondence between the circuit and the physical model is based on next premises:

a) The ground of the electric circuit - see node 0 in Fig. 2(b) - coincides with the ground terminal of the electric supply applied to the micromotor.

b) The input applied voltage in the active electric circuit terminal - see node 1 in Fig. 2(b) - coincides with the terminal of one of the six phases of the electric supply applied to the micromotor.

c) The output terminal voltage of the electrical circuit - see node 2 in Fig. 2(b) - coincides with the potential at the interface of the micromotor, which is the potential of the rotor. The interface is the surface of the resistive metal sheet of the rotor that is in contact with the air.

In the micromotor, the electrical resistance of the stator does not exist, and the inductive impedance is replaced by its dual capacitive impedance, which is $C_{1}$ in Fig. 2(b). The resistance and the inductive impedance of the rotor, referred to the stator, are replaced by their dual conductance $G_{r}{ }_{r}$ and capacitive impedance $C_{2}$, respectively. Conductance 
$G_{r}^{\prime}$ is expressed as:

$$
G_{r}^{\prime}=G_{2}^{\prime}+G_{2}^{\prime}\left(\frac{1}{S}-1\right)
$$

where $G_{2}^{\prime}$ is the conductance -resistance loss due to metallic resistive sheet of the rotor- and $S$ the slip of the micromotor. The slip $S$ is expressed as follows

$$
S=\frac{\omega / k_{n}-v}{\omega / k_{n}}
$$

where

$$
k_{n}=\frac{2 \pi}{\lambda_{n}}
$$

$\omega / k_{n}$ is the wave speed, $v$ the rotor velocity, and $\lambda_{n}$ is the length of each spatial harmonic.

The relationship between the lumped parametric equivalent circuit model and the physical nature of components of the micromotor is summarized as follows:

a) The capacitor $C_{1}$, placed in Fig. 2(b) between nodes 1 and 2 of the equivalent circuit, is the capacitance between two parallel plates separated by a distance, and air as dielectric material. These two planes contain the fixed and mobile elements of the electrostatic induction micromotor.

b) The capacitor $C_{2}$, placed in Fig. 2(b) between nodes 0 and 2 of the circuit, is the capacitance between the superior and inferior sheets of the mobile element of the electrostatic induction micromotor. Between these sheets there is a dielectric material, with a permittivity $\varepsilon_{\mathrm{b}}$.

c) The conductances $G_{2}^{\prime}$ and $G_{2}^{\prime}(1-S) / S$ are, respectively, the resistance losses due to the metallic resistive sheets of the rotor, and the mechanic power generated by the micromotor which is a function of $S$.

Fig. 3 illustrates that the results obtained using both the equivalent circuit model and Maxwell's equations coincide. Table II presents the values calculated for the components of the equivalent circuit.

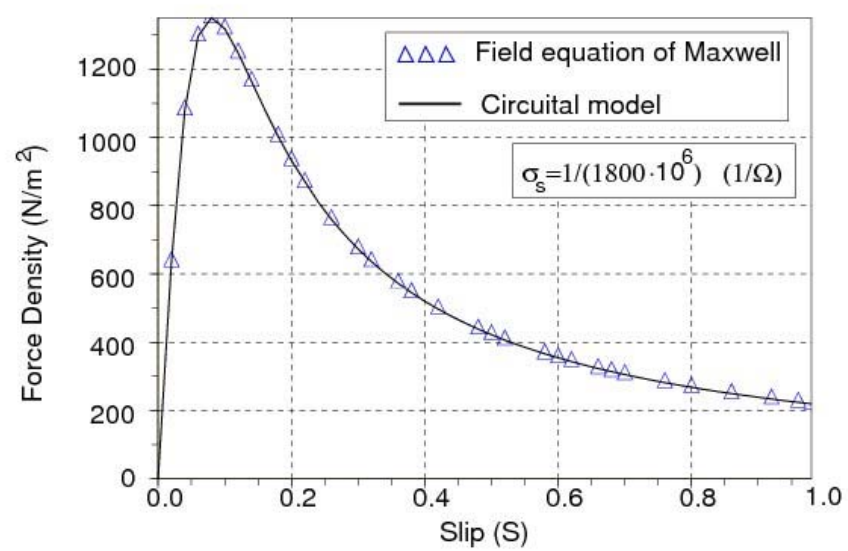

Fig. 3. Force Density vs. slip value for both the lumped parametric model and analytical Maxwell's field equation.
TABLE II

CALCUlated VAlues FOR LUMPED PARAMETERS PER PHASE

\begin{tabular}{|c|c|c|c|}
\hline$\sigma_{S}(1 / \Omega)$ & $\mathrm{C}_{1}(\mathrm{~F} / \mathrm{m})$ & $\mathrm{C}_{2}(\mathrm{~F} / \mathrm{m})$ & $\mathrm{G}_{2}^{\prime}\left(\Omega^{-1} / \mathrm{m}\right)$ \\
\hline $1 /\left(1800 \cdot 10^{6}\right)$ & $238.71857 \cdot 10^{-9}$ & $483.966 \cdot 10^{-9}$ & $9.4506 \cdot 10^{-1}$ \\
\hline
\end{tabular}

\section{SPATIAl HaRMONICS ANALYSIS}

In this section, we introduce the results of the spatial harmonics in three different zones at the time instant corresponding to half of the excited signal cycle. The zones are the stator (A), the air between the stator and the surface of the mobile part (B), and, finally, the surface of the mobile part (C). Fig. 4 illustrates these three zones. Then, we present the results obtained for the force density, taking into account the fundamental component and also the first harmonic. Fig. 5 shows the global results obtained for an analysis with FEM. The left part of Fig. (5) illustrates the potential distribution at the interface between rotor and stator in the micromotor and the right part shows the FEM mesh domain.

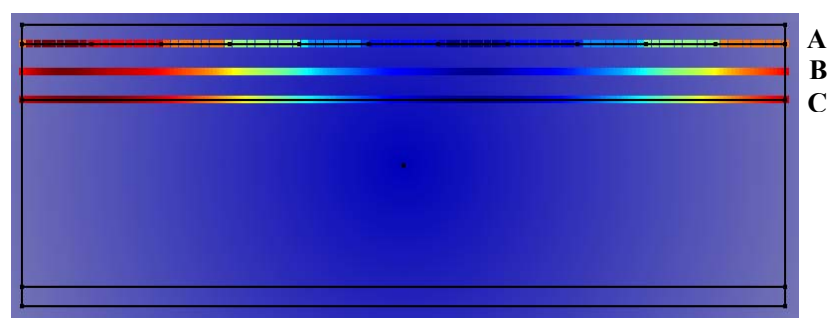

Fig. 4. Spatial harmonic analysis zones of the micromotor.

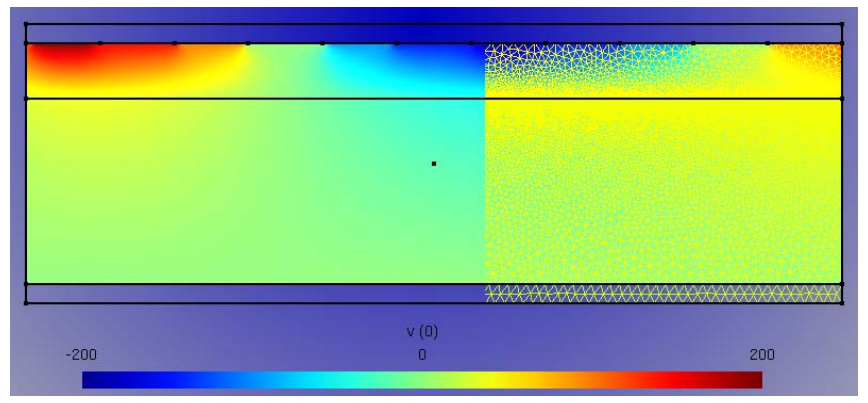

Fig. 5 Potential distribution and FEM mesh domain.

\section{A. Harmonics in the electrodes of the stator (zone A)}

Fig. 6 illustrates the potential spatial distribution in the electrodes of the micromotor at the time instant corresponding to the half of the excited signal cycle (named time instant T), in zone A (see Fig. 4).

Fig. 6 shows two different curves; the fundamental component - the first harmonic_-, and the addition of the first ten harmonics.

Fig. 7 illustrates the maximum potential amplitude distribution of the spatial harmonics. 


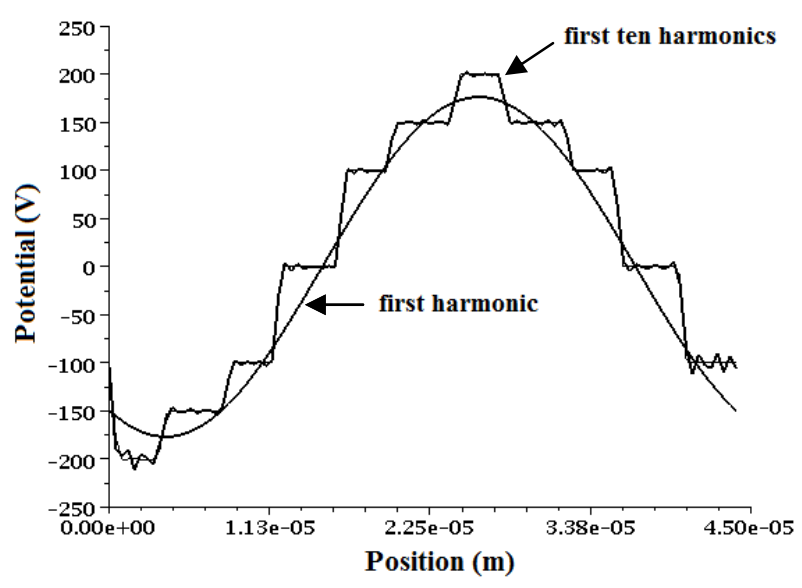

Fig. 6. Potential spatial harmonics in the stator electrodes (zone A).

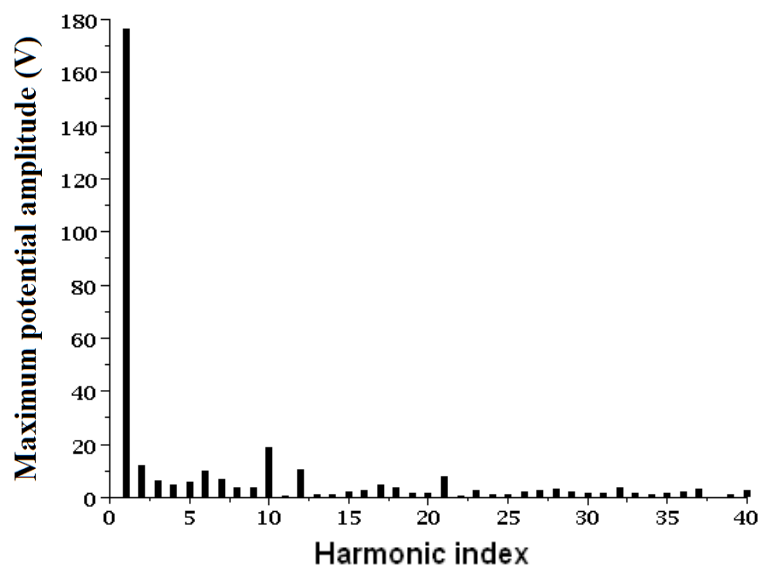

Fig. 7. Maximum potential amplitude of the harmonics in zone A.

\section{B. Harmonic in the air (zone B)}

Fig. 8 illustrates the potential distribution in the electrodes in zone B (see Fig. 4) at instant T. The wave is more smooth that the wave in zone A due to influence of the capacitive medium (filtering effect). The potential wave corresponding to the first harmonic it is also presented. Fig. 9 shows the harmonic distribution and potential magnitude for this region at the interface between rotor and stator. Comparison with Fig. 7 shows a reduction in spatial harmonics.

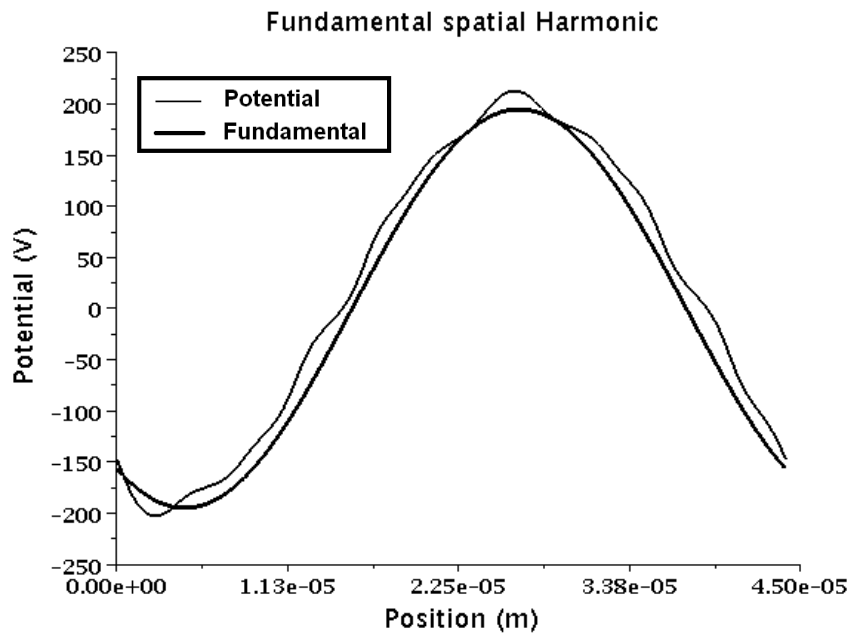

Fig. 8. Potential spatial harmonics in the air (zone B).

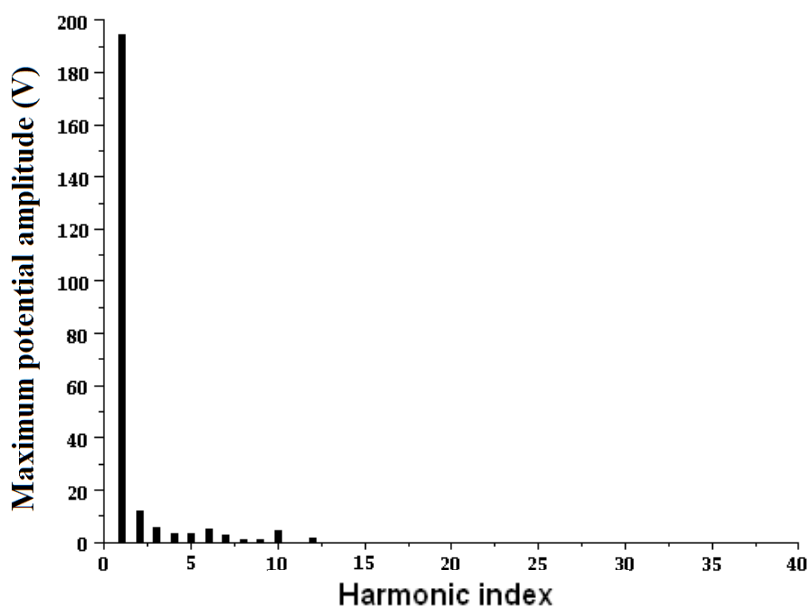

Fig. 9. Maximum potential amplitude of the harmonics in zone B.

\section{Harmonics in the interface (zone $C$ )}

This is the most important zone from a physical point of view. In this region, the induced charge is produced and the force density that drags the mobile part of the micromotor is generated. Fig. 10 illustrates the potential distribution in the surface of the rotor - see zone C in Fig. 4- at time instant T.

As Fig. 10 illustrates, the content in harmonics is much reduced due to the filter effect performed by the capacity elements and the superficial conductivity in the interface.

Fig. 11 presents a dramatic reduction in the number of harmonics in comparison with Fig. 7. They almost disappear and only the fundamental component remains.

\section{Force density harmonics in the in the interface (zone $C$ )}

To obtain the force density in the interface we use the lumped parametric equivalent circuit shown in Fig. 2. By applying the superposition principle, for each spatial harmonic, we calculate the respective force density as a function of the slip and Fig. 12 represents the second, third, fourth harmonics.

Fig. 13 illustrates the sum of the four first harmonics versus the fundamental for the force density in the interface. This is because the force density varies with the square of the magnitude of the harmonics, and, therefore, the effect of the harmonics is more negligible in the total force density.

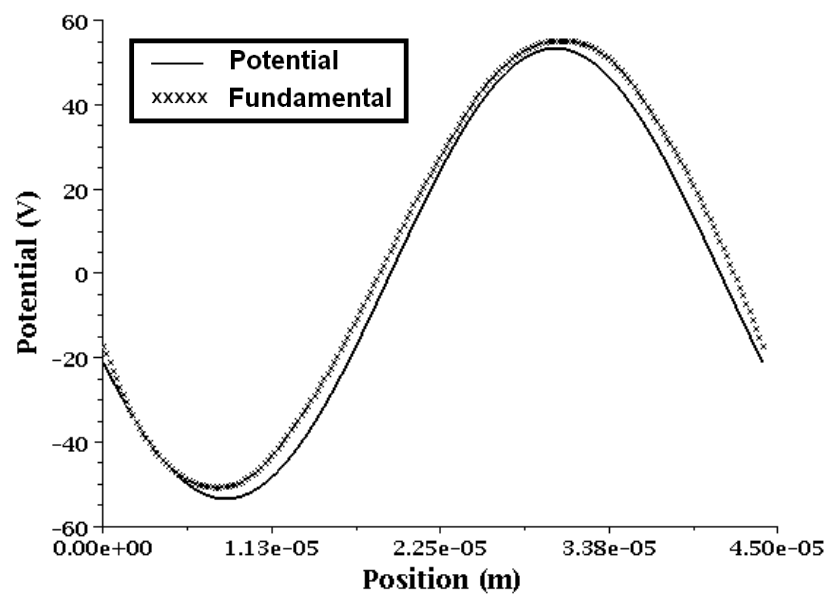

Fig. 10. Potential spatial harmonics in the surface of the mobile part of the micromotor (zone C). 


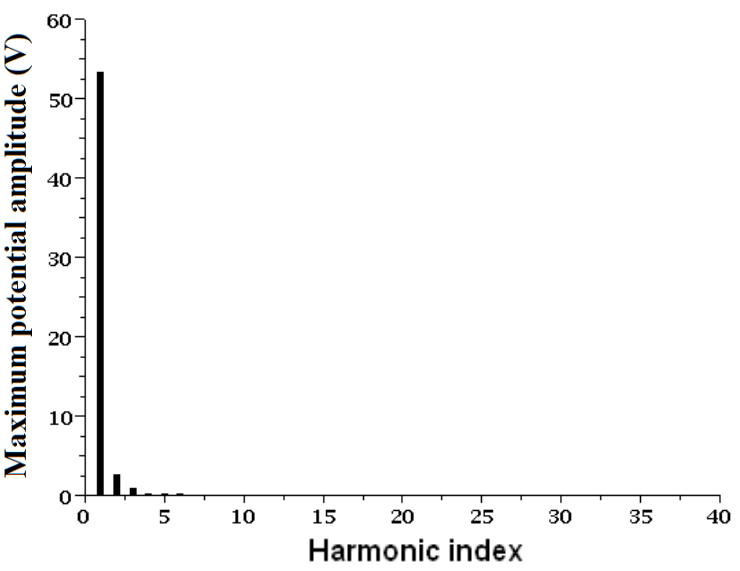

Fig. 11. Maximum potential amplitude of the harmonics in zone C.

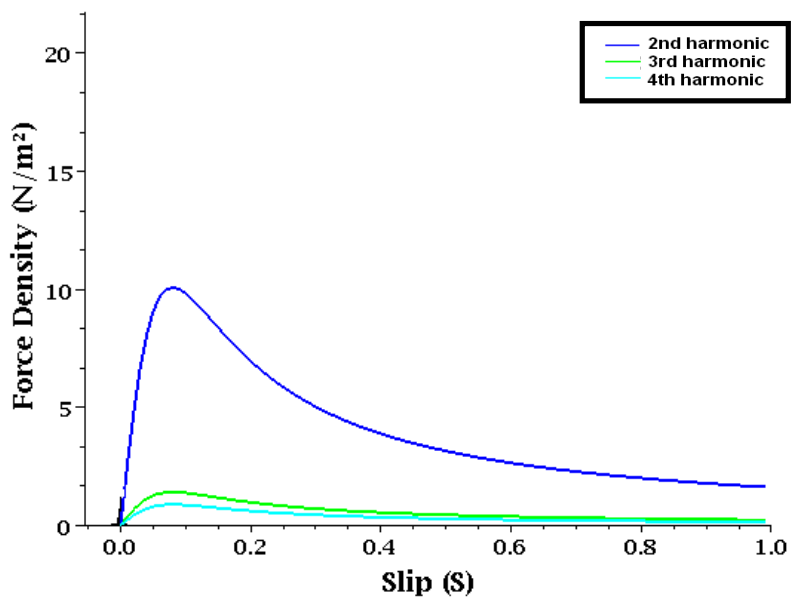

Fig. 12. Contributions to the force density of the $2^{\text {nd }}, 3^{\text {rd }}$ and $4^{\text {th }}$ harmonics.

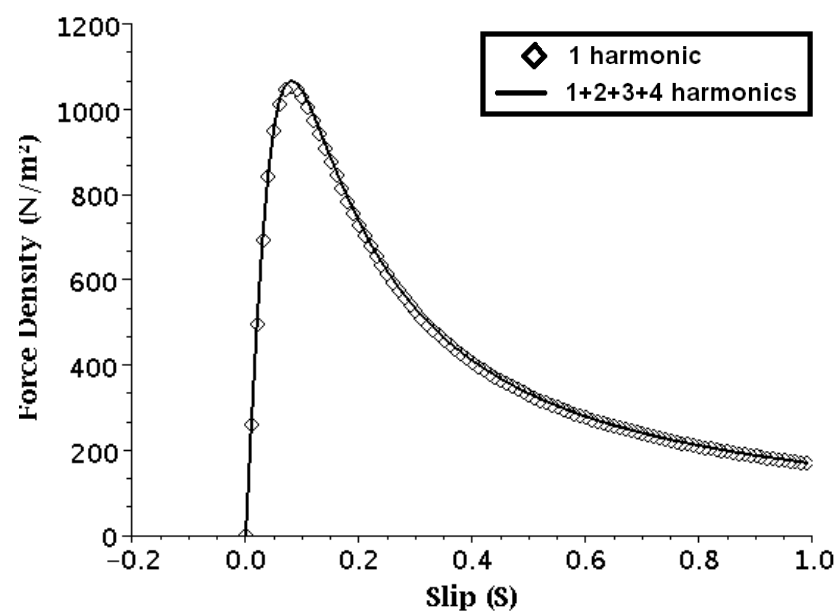

Fig. 13. Force density due to the fundamental harmonic and the first harmonics.

\section{CONCLUSION}

We have obtained spatial harmonics of the excitation potential signal in three zones between the rotor and stator of a polyphase electrostatic induction micromotor. From potential spatial harmonics analysis on each electrode, we obtain spatial harmonics on force density function. We have used the parametric equivalent circuit [7], and demonstrated that the voltage spatial harmonics are attenuated because the filtering characteristic and superficial conductivity in the interface. Because the force density is a function of the voltage harmonic square, the spatial harmonics of the force density are dramatically attenuated. Currently, we are designing an electrostatic microswitch prototype as a previous test of a future micromotor prototype.

\section{REFERENCES}

[1] T.C. Neugebauer, D.J. Perreault, J. F. Lang, and C. Livermore, "A sixphase multilevel inverter for MEMS electrostatic induction micromotor," IEEE Trans. Circuits Syst. II, Exp. Briefs, vol. 51, no.2, pp. 49-56, Feb. 2004.

[2] S. Bart and J. Lang, "Electroquasistatic induction micromotors," in Proc. IEEE Micro Electro Mechanical Systems Workshop '89, Salt Lake City, UT, 1989, pp. 7-12.

[3] F. J. Santana, J. M. Monzón, S. García-Alonso and J. A. MontielNelson, "Analysis and Modeling of an Electrostatic Induction Micromotor", in Proc. of the IEEE ICEM-08, Villamoura, Portugal, Sept. 2008.

[4] L.G. Frechette, S.F. Nagle, R. Ghodsi, S.D. Umaus, M.A. Schmidt, J.H. Lang, "An electrostatic induction micromotor supported on gaslubricated bearings", IEEE 14th International Conference, MEMS 2001, January 2001, Interlaken, Switzerland.

[5] P. Krein, "Analysis of corona motors and micro motors by means of effective gap conductivity", IEEE Trans. Ind. Appl. 31 (4) (1995) $752-760$.

[6] E. A. Klingshirn, H. E. Jordan, "Polyphase Induction Motor Performance and Losses on Nonsinusoidal Voltage Sources," IEEE Transactions on Power Apparatus and Systems, vol. PAS 87, NO. 3, pp. 624-631, March, 1968.

[7] F. J. Santana, J. M. Monzón, S. García-Alonso, J. A. Montiel-Nelson, "Lumped parametric model for an electrostatic induction micromotor using GA's methods". in Proc. of ISEF 2009 - XIV International Symposium on Electromagnetic Fields in Mechatronics, Electrical and Electronic Engineering. Arras, France, September 10-12, 2009.

[8] P. Dular, C. Geuzaine, F Henrotte and W. Legros, "A General Environment for the Treatment of Discrete Problems and its Application to the Finite Element Method", IEEE Transactions on Magnetics, vol. 34, pp. 3395-98, Sep. 1998.

[9] A. Nicolet and F. Delincé "Implicit Runge-Kutta Methods for Transient Magnetic Field Computation", IEEE Transactions on Magnetics, vol. 32, n. 3, 1996.

[10] C. Geuzaine and J. F. Remacle, "Gmsh: a Three-Dimensional Finite Element Mesh Generator with Built-in Pre-and Post-Processing Facilities", Int. J. for Numerical Methods in Engineering, May 2009.

\section{BIOGRAPHIES}

F. J. Santana-Martín studied, both, Technical Industrial Engineering and Electronic Engineering. Assistant Professor of Circuit Theory in the University of Las Palmas de Gran Canaria, ULPGC, (Spain). Member of the Micromechanical Systems Division of the Institute for Applied Microelectronics (IUMA), develops, his research activities in the field of MEMS.

J. M. Monzón-Verona. Ph. D. in Industrial Engineering from the University of University of Las Palmas de Gran Canaria, ULPGC, Spain. He is Titular Professor at the Industrial Engineering School and professor of Circuit Theory en el Electric Engineering Department of the ULPGC. Member of the Micromechanical Systems Division of the Institute for Applied Microelectronics (IUMA) His current research fields include MEMS.

S. García-Alonso. Ph. D. in Industrial Engineering from the University of Navarra in San Sebastián, Spain. He has been working with industrial companies in their technical departments in different sectors from 1997 to 2001. Then, he joined LABEIN, a technological research \& innovation centre, settled in Bilbao, Spain. Currently, since 2005, he is member of the Department of Electronic and Automation Engineering at the University of Las Palmas de Gran Canaria, ULPGC, Spain, and focuses his research in MEMS, in the Micromechanical Systems Division of the Institute for Applied Microelectronics (IUMA). 
J. A. Montiel-Nelson received the M.S. degree in electrical engineering and the Ph.D. degree from the University of Las Palmas de Gran Canaria, Gran Canaria, Spain, in 1991 and 1994, respectively. From 1996 to 1997, he was a Visiting Scientist at the Centre for Very High Speed Microelectronic Systems of Edith Cowan University, Western Australia, Australia, and in the Department of Computer and Communication Engineering of the same university. From 1997 to 2003, he was a Titular Professor in the Department of Electronic Engineering at the University of Las Palmas de Gran Canaria, where he is currently Full Professor. Since 1990, he has been with the Institute for Applied Microelectronics (IUMA) working on CAD and VLSI design of emergent technologies, performance estimation and optimization of circuits and systems, and functional and formal verification. He has authored or coauthored more than 50 papers in edited books, international journals, and conference proceedings. His current research fields include very high-speed integrated circuit (VHSIC) design (circuit, logic, and module design; full-custom design and ASIC synthesis) in GaAs, SiGe, InP, and very deep submicrometer CMOS technologies; performance estimation and optimization of digital integrated circuits and systems (including power delay tradeoff, timing, and interconnect analysis); and verification of circuits and systems (satisfiability, functional verification, and formal checking). 